\title{
Conditions for substorm onset by the fast reconnection mechanism
}

\author{
M. Ugai \\ Research Center for Space and Cosmic Evolution, Ehime University, Matsuyama 790-8577, Japan \\ Received: 6 August 2008 - Revised: 17 September 2008 - Accepted: 20 October 2008 - Published: 2 December 2008
}

\begin{abstract}
The fast reconnection mechanism, involving slow shocks and Alfvénic fast plasma jets, is most responsible for the explosive conversion of magnetic energy associated with geomagnetic substorms and solar flares. In this paper, the spontaneous fast reconnection model is applied to well-known phenomena of substorms. When the east-west width of the tail current sheet becomes 3-4 times larger than its north-south thickness, the fast reconnection mechanism can fully be established, which may lead to substorm onset. The resulting Alfvénic jet can exactly explain, both qualitatively and quantitatively, the in-situ satellite observations of the traveling compression regions (TCRs) associated with large-scale plasmoids propagating down the tail. Also, the earthward fast reconnection jet causes drastic magnetic field dipolarization, so that the sheet current ahead of the magnetic loop of closed field lines suddenly turns its direction toward the loop footpoint and a large-scale current wedge is formed according to the growth of field-aligned currents. It is demonstrated that an MHD generator arises ahead of the magnetic loop and drives the current wedge to distinctly enhance the current density in a pair of thin layers of the loop footpoint, giving rise to drastic heating in the form of two ribbons.
\end{abstract}

Keywords. Magnetospheric physics (Storms and substorms) - Solar physics, astrophysics, and astronomy (Flares and mass ejections) - Space plasma physics (Magnetic reconnection)

\section{Introduction}

The fast reconnection mechanism, involving Alfvénic plasma jets, must be most responsible for flare phenomena in space plasmas (Shibata, 1999; Sharma et al., 2008). Then, question is how the fast reconnection mechanism can be re-

Correspondence to: M. Ugai

(ugai@cosmos.ehime-u.ac.jp) alized as an eventual solution of MHD equations in hightemperature plasmas of extremely large magnetic Reynolds number. A possible fast reconnection configuration was first proposed by Petschek (1964) on the basis of two-dimensional (2-D) steady MHD equations, and it was supposed that the diffusion region size would be controlled by external boundary conditions. Hence, most of the theoretical studies have been directed to looking for such a fast reconnection solution that can be realized without significant influence of plasma resistivity (Vasyliunas, 1975; Priest and Forbes, 1986).

On the other hand, it was demonstrated for the first time that the fast reconnection mechanism can be realized when a localized resistivity is applied (Ugai and Tsuda, 1977). Hence, we have proposed the spontaneous fast reconnection model and demonstrated by 2-D and three-dimensional (3-D) simulations that the fast reconnection mechanism can be realized as an eventual solution of MHD equations by the nonlinear instability due to positive feedback between current-driven anomalous resistivities and global reconnection flows (Ugai, 1984, 1999; Ugai and Zheng, 2005). In fact, if there is no effective resistivity, extreme current sheet thinning occurs because of nonlinear pinch effects (Ugai, 1986, 2008b), which may cause anomalous resistivities due to current-driven microturbulences (Lui, 2001). Since the fast reconnection evolution is so poweful, we have called this process "spontaneous reconnection", but it might also be called "undriven reconnection" (Daughton et al., 2006).

Many MHD models have assumed explicit resistivity models, such as current-driven anomalous resistivities, in 3-D (Raeder, 2003; Isobe et al., 2005) and 2-D (Uzdensky, 2003; Klimas et al., 2004; Yokoyama and Shibata, 1994) situations. In general, these studies have shown that if the resistivity is enhanced locally around an X point, magnetic reconnection occurs effectively, which is consistent with our results. In this paper, we show how the spontaneous fast reconnection model can be applied to well-known substorm and flare signatures.

Published by Copernicus Publications on behalf of the European Geosciences Union. 


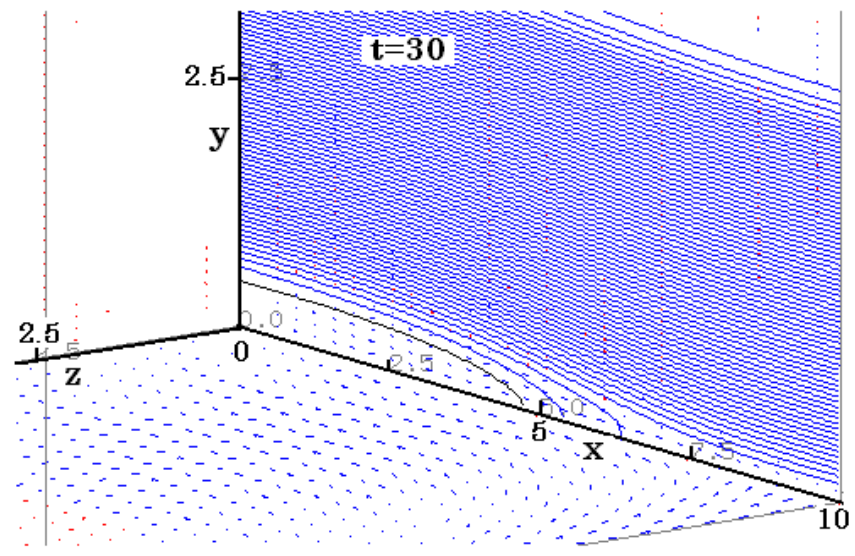

Fig. 1. Magnetic field and plasma flow configuration for the uniform resistivity model with $\eta=0.001$.

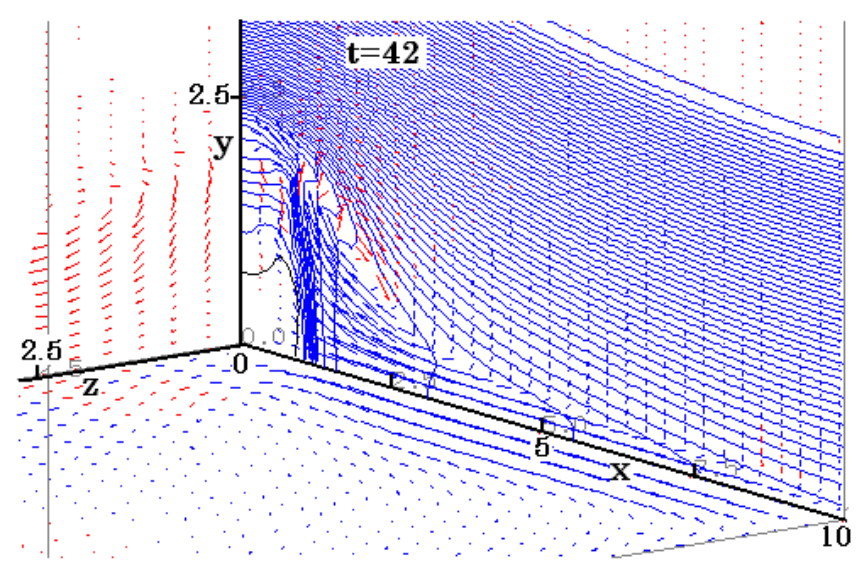

Fig. 2. Magnetic field and plasma flow configuration for the anomalous resistivity model (Eq. 4) with $V_{C}=12$, where the velocity scale is the same as in Fig. 1.

\section{Simulation modeling}

The compressible MHD equations are

$D \rho / D t=-\rho \nabla \cdot \mathbf{u}, \quad \rho D \mathbf{u} / D t=-\nabla P+\mathbf{J} \times \mathbf{B}$,

$\partial \mathbf{B} / \partial t-\nabla \times(\mathbf{u} \times \mathbf{B})=-\nabla \times(\eta \mathbf{J})$,

$\rho D e / D t=-P \nabla \cdot \mathbf{u}+\eta \mathbf{J}^{2}$,

$\mathbf{J}=\nabla \times \mathbf{B} / \mu_{0}, \quad \nabla \cdot \mathbf{B}=0$,

where $D / D t \equiv \partial / \partial t+\mathbf{u} \cdot \nabla$; the gas law, $P=(\gamma-1) \rho e$, is assumed $[e$ is the internal energy per unit mass, and $\gamma$ is the specific heat ratio with $\gamma=5 / 3$ assumed here (an adiabatic case)], as is Ohm's law, $\mathbf{E}+\mathbf{u} \times \mathbf{B}=\eta \mathbf{J}$ ( $\eta$ may be an effective resistivity).

An antiparallel magnetic field $\mathbf{B}=\left[B_{x}(y), 0,0\right]$ is initially assumed as: $B_{x}(y)=\sin (\pi y / 2)$ for $0<y<1 ; B_{x}=1$ for $1<y<4 ; B_{x}=\cos [(y-4) \pi / 1.2]$ for $4<y<4.6 ; B_{x}=0$ for



Fig. 3. Magnetic field and plasma flow configurations for the anomalous resistivity model (Eq. 4) with $V_{C}=12$ in an open system; in the upper panel, the plasmoid is shown by the isosurface of plasma pressure.

$y>4.6$; also, $B_{x}(y)=-B_{x}(-y)$ for $y<0$. Fluid velocity $\mathbf{u}=(0,0,0)$, and plasma pressure $P(y)$ satisfies the pressurebalance condition,

$P+B_{x}^{2}=1+\beta_{0}$,

where $\beta_{0}$ is the ratio of the plasma pressure to the magnetic pressure in the ambient magnetic field region $1<y<4$, so that $P(y=0)=1+\beta_{0}$ initially $\left(\beta_{0}=0.15\right.$ is taken throughout the present studies). Also, plasma density $\rho$ initially satisfies

$\rho=P /\left(1+\beta_{0}\right)$.

The normalization of quantities, based on the initial quantities, is self-evident: Distances are normalized by the current sheet half-thickness $d_{0}$, B by $B_{x 0}, P$ by $P_{0}=B_{x 0}^{2} /\left(2 \mu_{0}\right)$; also, $\rho$ by $\rho_{i}=\rho(y=0)$, u by $V_{A x 0}\left(=B_{x 0} / \sqrt{\mu_{0} \rho_{i}}\right)$, time $t$ by $d_{0} / V_{A x 0}$. The Alfvén velocity in the ambient magnetic field region $(1<y<4)$ is given by $V_{A e}=V_{A x 0} / \sqrt{\rho_{e}} \sim 2.7$ $\left[\rho_{e}=\beta_{0} /\left(1+\beta_{0}\right)\right]$, and the normalized temperature is given by $T=P / \rho=1+\beta_{0}$. Here, the conventional symmetry boundary conditions are assumed on the $z=0, x=0$ and $y=0$ planes. Hence, the computational region can be restricted to the first quadrant only and taken to be a rectangular box, $0 \leq x \leq L_{x}, 0 \leq y \leq L_{y}$, and $0 \leq z \leq L_{z}$.

The effective resistivity for the Ohm's law (normalized by $\mu_{0} d_{0} V_{A x 0}$ ) should be provided by current-driven anomalous resistivities in space plasmas and may be assumed as,

$$
\begin{aligned}
\eta_{D}(\mathbf{r}, t) & =k_{d}\left[V_{d}(\mathbf{r}, t)-V_{C}\right] \quad \text { for } V_{d}>V_{C}, \\
& =0 \quad \text { for } V_{d}<V_{C},
\end{aligned}
$$



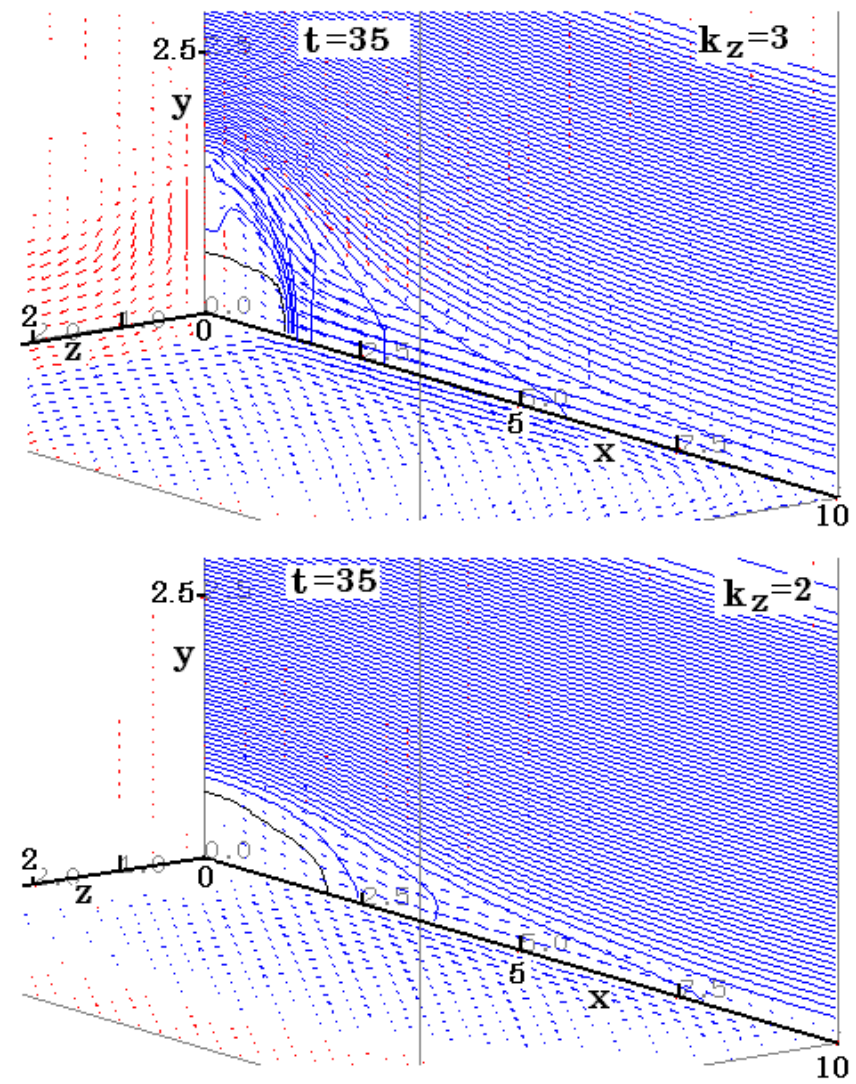

Fig. 4. Magnetic field and plasma flow configuration for the anomalous resistivity model (Eq. 4) with $V_{C}=4$ for the cases $k_{z}=2$ and 3 in Eq. (5).

where $V_{d}(\mathbf{r}, t)=|\mathbf{J}(\mathbf{r}, t) / \rho(\mathbf{r}, t)|$ is the relative electron-ion drift velocity and $V_{C}$ the threshold (here, $k_{d}=0.003$ is taken).

In order to disturb the initial configuration, a localized resistivity is assumed at $x=d_{x}$,

$\eta(\mathbf{r})=\eta_{0} \exp \left[-\left[\left(x-d_{x}\right) / k_{x}\right]^{2}-\left(|y| / k_{y}\right)^{3}-\left(|z| / k_{z}\right)^{3}\right]$,

which provides a seed of magnetic reconnection. Here, we take $\eta_{0}=0.02$ and $k_{x}=k_{y}=0.8$. This reconnection disturbance is imposed only in the initial time range $0<t<4$, and the resitivity model will be assumed for $t>4$.

The numerical procedure for solving the full MHD equations is simple and the same as in our earliest papers (Ugai, 1988, 2008b). Throughout our reconnection studies, the numerical parameters have been carefully chosen, so that the numerical resistivity is much smaller than the physical resistivity (Ugai, 2008b). Here, the mesh sizes are taken to be $\triangle x=0.04, \triangle y=0.015$, and $\triangle z=0.1$ or $\triangle z=0.05$.

\section{Spontaneous fast reconnection model}

If there is no dissipation mechanism (effective resistivity), initiated by the reconnection disturbance (Eq. 5), drastic current sheet thinning occurs, leading to extreme increase in

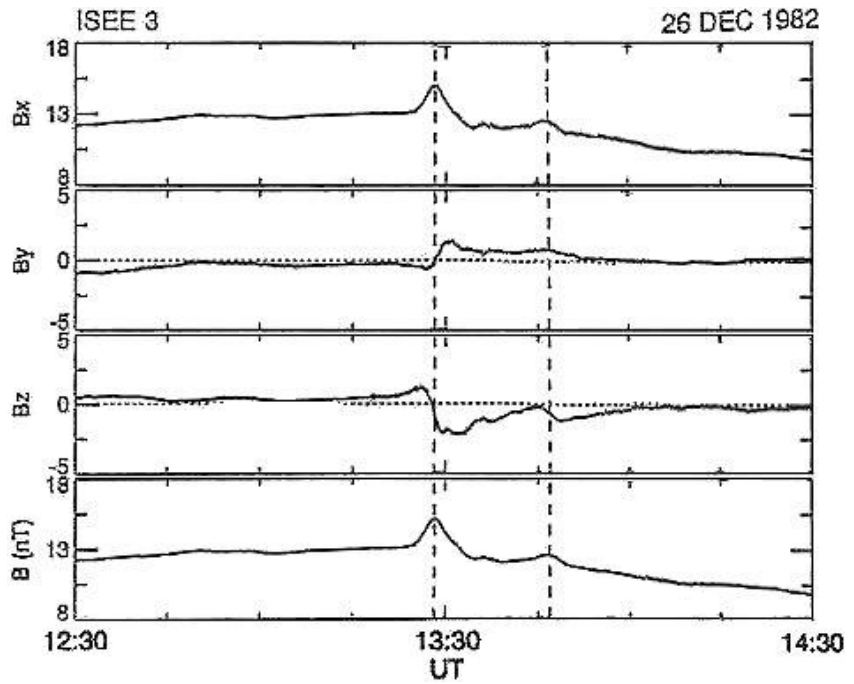

Fig. 5. ISEE 3 magnetic field observations taken in the north lobe of the tail at $x=-73 R_{E}$ in GSM coordinates (after Slavin et al., 1993).

the current density (Ugai, 1986, 2008b). If there is an effective resistivity, the current density increase around the $\mathrm{X}$ point may be suppressed, and magnetic reconnection builds up. We have demonstrated that the reconnection process is strongly influenced by the resistivity model (Ugai, 1992). For the Spitzer resistivity model, the resistivity becomes reduced at the $\mathrm{X}$ point because of the temperature increase by the reconnection, so that the associated reconnection is less effective than that for the uniform resistivity model (Ugai, 1992; Ugai and Zheng, 2005).

First, let us take the uniform resistivity model with $k_{z}=5$ and $d_{x}=L_{x}=10$ in Eq. (5) $(\triangle z=0.1)$, and the symmetry boundary condition is imposed on the $x=10$ plane. In this case, no explicit numerical dissipation term is needed, and magnetic reconnection starts immediately for $t>4$. Figure 1 shows the resulting magnetic field and plasma flow configuration and indicates that no effective reconnection occurs. In what follows, some numerical dissipations must be introduced for the fast reconnection mechanism, and the associated numerical resistivity $\eta_{N}$ can be estimated by examining the numerical reconnection with zero physical resistivity. Then, we find that it works like a uniform resistivity with $\eta_{N} \sim 0.0005$ for the present parameter values (Ugai, 2008b).

For the anomalous resistivity model (Eq. 4) with $V_{C}=12$, once the threshold is exceeded, both the reconnection flow and the anomalous resistivity drastically grow to enhance each other, eventually leading to the fast reconnection mechanism. Figure 2 shows the resulting fast reconnection configuration, where the outflow velocity has been accelerated to the Alfvén velocity because of standing slow shocks. In this case, the reconnection jet is blocked by the wall boundary (at $x=0$ ), leading to magnetic field dipolarization and formation of a large-scale magnetic loop of closed field lines. 


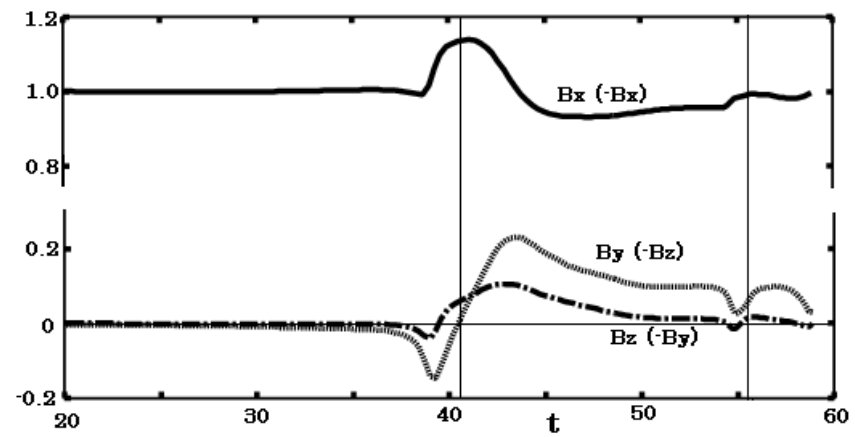

Fig. 6. Temporal variations of magnetic fields observed by virtual satellites located in the magnetic field (low- $\beta$ ) region, where $B_{x}, B_{y}$ and $B_{z}$ in the simulation coordinates correspond to $-B_{x},-B_{z}$ and $-B_{y}$, respectively, in GSM coordinates.

On the other hand, if the outflow jet can freely flow in an open system, a large-scale plasmoid is formed ahead of the jet and propagates along the magnetic field (Ugai, 1989, 1995). Hence, when the $x=L_{x}=20$ plane is assumed to be the free boundary with $d_{x}=0$ in Eq. (5), Fig. 3 shows the resulting 3-D plasmoid structure, inside which the plasma pressure is notably enhanced (Ugai and Zheng, 2005). Note that unlike the conventional plasmoid magnetic field lines are not necessarily closed inside the plasmoid, since this plasmoid is driven and sustained by the Alfvénic fast reconnection jet.

The fast reconnection mechanism can fully be realized when the $z$ directional current sheet width is 3-4 times larger than its $y$ directional thickness (Ugai, 2007a). Hence, it may be instructive to see the reconnection processes for $k_{z} \leq 3$ in Eq. (5) with the other conditions being the same as in Fig. 2 except that $V_{C}=4$ in Eq. (4) and $\Delta z=0.05$. Figure 4 shows the resulting configurations; for $k_{z}=2$, no effective reconnection occurs, since the outflow jet can hardly be accelerated. For $k_{z}=3$, the reconnection jet is considerably accelerated but soon redued before it attains the Alfvén velocity. For these cases, the ambient magnetized plasma cannot effectively collapse into the $\mathrm{X}$ region, so that the fast reconnection mechanism cannot be realized (Ugai, 2008b).

If a thin current sheet of sufficiently large scale is formed, initiated by a reconnection disturbacne, current sheet thinning drastically occurs to distinctly enhance the current density. For simulation studies, the enhanced currents may be suppressed by effective physical and/or numerical resistivities. Hence, in performing reconnection simulations, it is essential to relevantly choose the parameter values so that the numerical resistivity is much smaller than the physical resistivity. Throughout our MHD simulations, the numerical resistivity, estimated to be $\sim 0.0005$, is found to be much smaller than the physical (anomalous) resistivity (Ugai, 2008b).

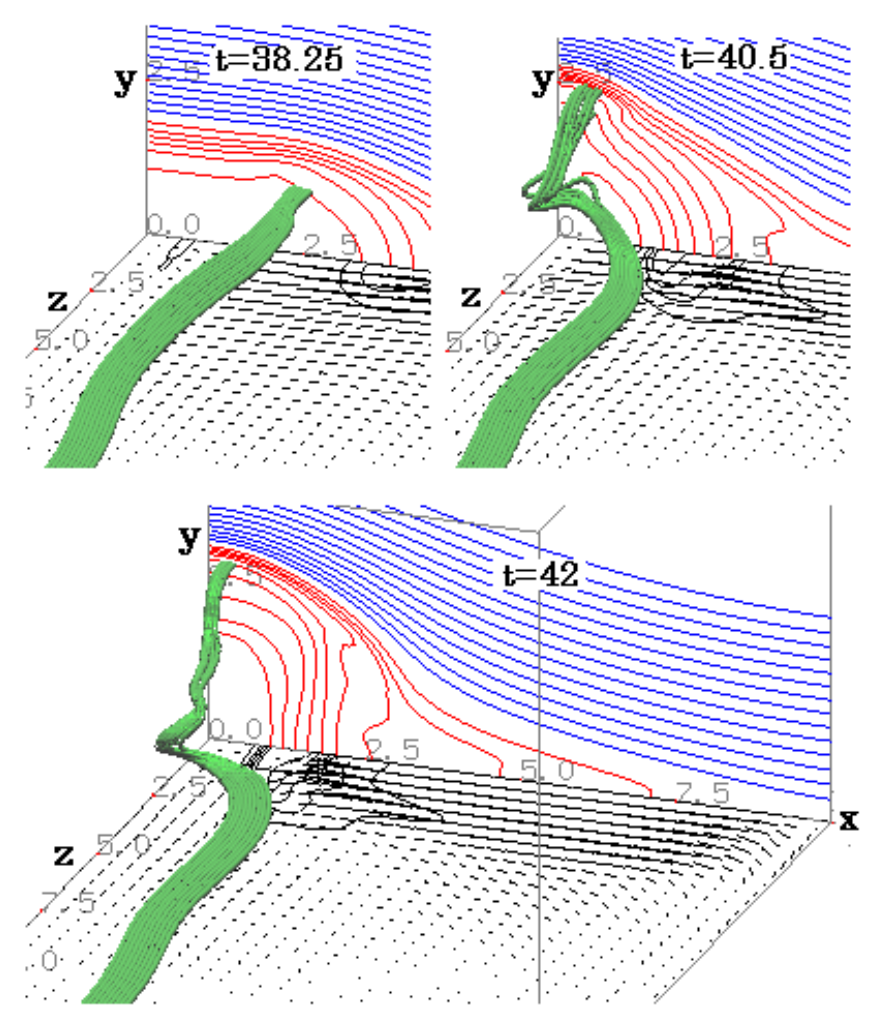

Fig. 7. Current flow lines starting from the segment, $2.16<x<2.72$ at $y=0.27$ and $z=13$ for the case of $k_{z}=7$ in Eq. (5).

\section{Applications to substorms and flares}

The spontaneous fast reconnection model may be applied to well-known phenomena observed in geomagnetic substorms and solar flares. Since many distinct phenomena characterizing substorms and flares are associated with macroscopoic quantities, they should be explained by MHD equations.

\subsection{Traveling compression regions}

It is well known by satellite observations that according to substorm onset a large-scale plasmoid is formed and propagates down the geomagnetic tail, and as a clear evidence of substorm, the so-called traveling compression regions (TCRs) are observed. Slavin and his colleague have systematically studied TCR events for many years, and Fig. 5 shows the typical observed magnetic field variations, which indicate the pulse-like compression of the earthward $\left(B_{x}\right)$ field component as well as the northward to the southward tilting of the $B_{z}$ field component (Slavin et al., 1993). This TCR signature, observed in the distant tail lobe region, has been expected to be associated with a plasmoid bulge passing through the plasma sheet down the tail. The TCR signature is very simple and clear, but the previous theoretical studies could not succeed in explaining it quantitatively. 

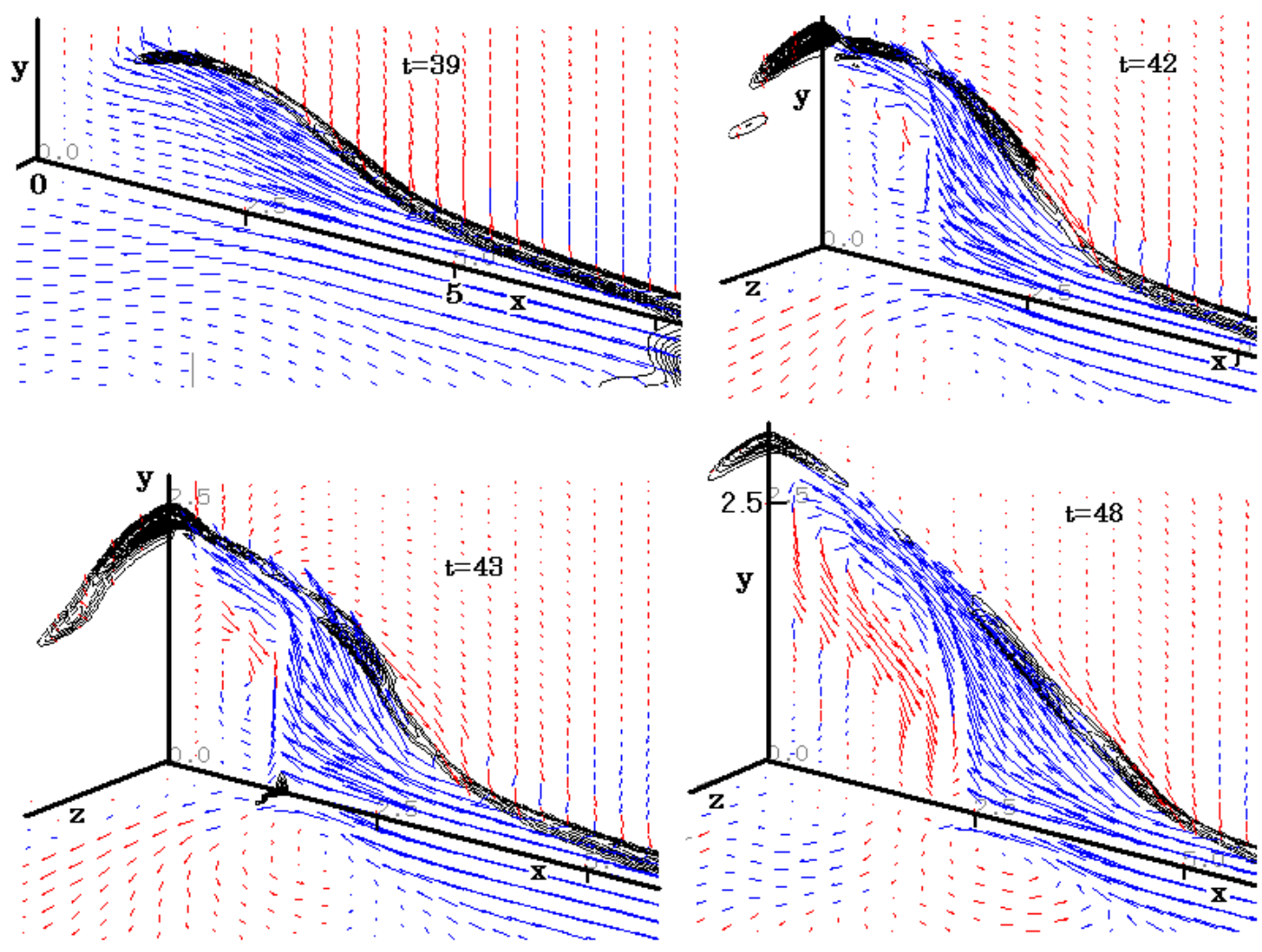

Fig. 8. Plasma flow vectors and distributions of the joule heating $\eta \mathbf{J}^{2}$.

The spontaneous fast reocnnection model in an open system (Fig. 3) can readily be applied to the TCR signatures (Ugai and Zheng, 2006a, b), so that Fig. 6 shows the temporal behaviors of the magnetic field components observed by a virtual satellite located in the ambient magnetic field (lobe) region in the simulation box (Fig. 3). We readily see that the temporal field variations observed by the virtual satellite are, both qualitatively and quantitatively, in good agreement with the TCR signatures. We hence conclude that the TCR observations provide a definite evidence that the fast reconection mechanism is in fact realized in the tail current sheet during substorms.

\subsection{Substorm current wedge}

If the fast reconnection builds up somewhere in the magnetotail, it should extend in both the earthward and tailward directions. In fact, in association with substorms, distinct plasma processes are observed in the near-Earth magnetotail, such as strong earthward jet and magnetic field dipolarization. In particular, it has long been believed that the near-Earth tail sheet current is suddenly disrupted so that a large-scale current wedge evolves and links the tail sheet current to the auroral electrojet through field-aligned currents (McPherron et al., 1973). Since there have been many observational evidences supporting this idea, it is essential for substorm stud- ies to clarify the physical mechanism of current wedge evolution.

For this long-standing question, we take $d_{x}=L_{x}=10$ in Eq. (5), and the symmetry boundary condition is imposed on the $x=L_{x}$ boundary for the resistivity model (Eq. 4) with $V_{C}=12$ (Ugai and Kondoh, 2006; Ugai, 2007a). We then find that when the fast reconnection jet collides with the magnetic loop of closed field lines, field-aligned currents suddenly appear because of drastic increase in the sheared field $B_{z}$ inside the loop, leading to drastic evolution of the current wedge. Figure 7 typically shows the current flow lines for the case of $k_{z}=7$ in Eq. (5) and indicates that the current, initially flowing into the current sheet, suddenly turns its direction toward the loop footpoint, where currents are locally concentrated and intensified.

The key to the current wedge evolution is the 3-D Alfvénic fast reconnection jet limited in the finite extent in the zdirection, and the underlying physical mechanism is consistent with the physical picture of the substorm current wedge. According to the current wedge evolution, the concentrated currents may give rise to joule heating in the layer of the loop footpoint. However, the current wedge evolution (Fig. 7) does not exactly model the substorm current wedge, since the $x=0$ boundary does not model the realistic ionopheric boundary; in fact, the plasma situations in the ionosphere are much more complicated. Hence, it may be instructive 

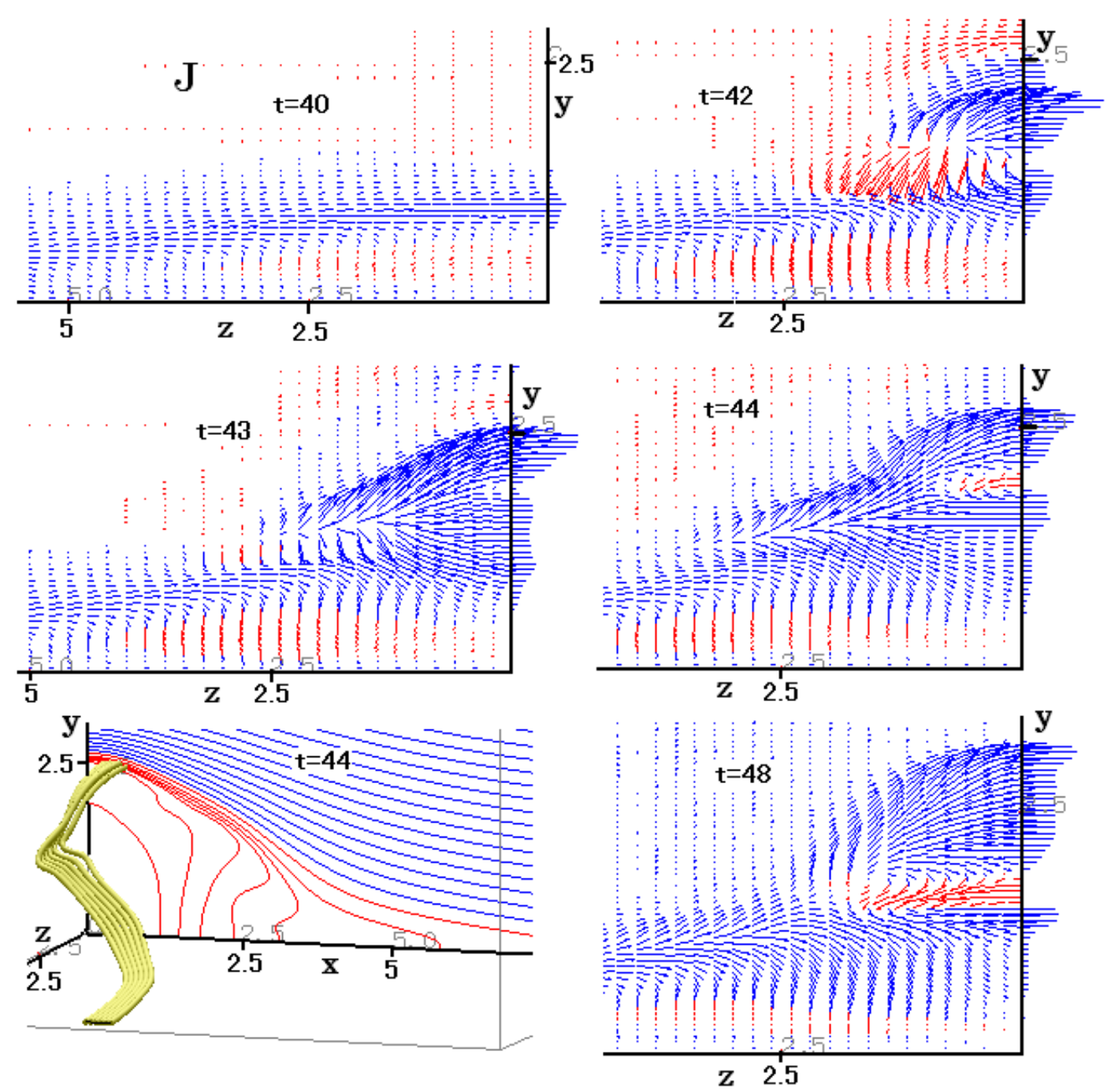

Fig. 9. Current density vectors at different times in the chromosphere $(x=0)$. In the left bottom, the current wedge formation is shown.

to model the solar surface and examine the basic effects of the current wedge evolution.

\subsection{Two-ribbon flares}

The solar surface may be modeled as follows (Ugai, 2007b, 2008a): Plasma density $\rho$ initially satisfies

$\rho(x, y)=\left\{R_{0} \exp \left[-(x / 0.4)^{4}\right]+1\right\} P(y) /\left(1+\beta_{0}\right)$,

where $R_{0}=100$ is taken, and the $x=0$ plane corresponds to the chromosphere, and the corona is for $x \gg 0.4$. Also, in addition to the resistivity model (4), we may take another anomalous resistivity model

$$
\begin{aligned}
\eta_{J}(\mathbf{r}, t) & =k_{j}\left[|\mathbf{J}(\mathbf{r}, t)|-J_{C}\right]^{n} \quad \text { for }|\mathbf{J}|>J_{C} . \\
& =0 \quad \text { for }|\mathbf{J}|<J_{C},
\end{aligned}
$$

where $n=2, J_{C}=3$ and $k_{j}=0.0001$ are assumed. Hence, we may generally take the resistivity model,

$\eta=\eta_{S}+\eta_{D}+\eta_{J}$

where $\eta_{S}=\eta_{C}\left(T / T_{0}\right)^{-1.5}$ is the Spitzer resistivity $(T=P / \rho$ is the temperature and $T_{0}=1+\beta_{0}$ the initial coronal temperature; also, $\eta_{C}=0.0000001$ is assumed). The other conditions are taken to be quite the same as in Sect. 4.2.

Figure 8 shows the plasma flow vectors and the distributions of the joule heating $\eta \mathbf{J}^{2}$ at different times, and Fig. 9 shows the temporal changes of the current density vectors in the chromosphere $(x=0)$. According to the current wedge evolution, the current density is suddenly enhanced in the chromosphere, leading to distinct increase of the joule heating in the chromospheric thin layer around the separatrix (Karlicky, 1995). In the corona $(x>1)$, the joule heating along the magnetic loop boundary (Fig. 8) corresponds to the standing slow shock layer. The strong joule heating in the 


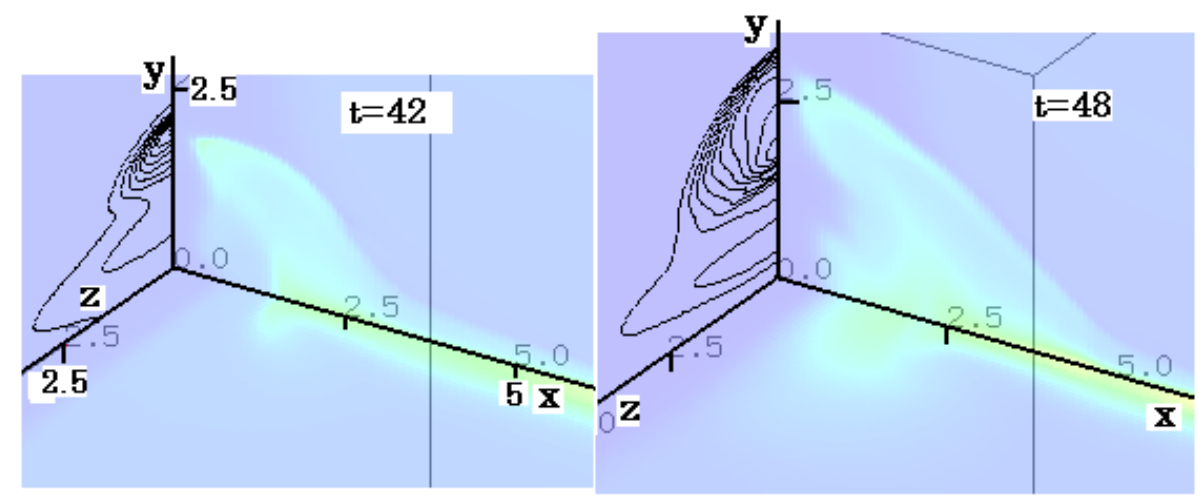

Fig. 10. Temperature distributions in the corona and in the chromosphere $(x=0)$ by contour lines at different times.

chromosphere may enhance the temperature. Hence, Fig. 10 shows the temperature distributions and indicates that the chromospheric temperature becomes more than 20 times its initial value.

We see from Fig. 10 that the chromospheric temperature is suddenly enhanced in the shape of two ribbons (due to symmetry boundaries), and the chromospheric region of enhanced temperature becomes wider and shifts upward with time. Also, the flare loop of high temperature expands outward in the corona, and the significant chromospheric evaporation occurs (Fig. 8) because of the pressure enhancement in the chromosphere. These features are consistent with the well-known morphological features of two-ribbon flares.

Then, an important question is how the current wedge can build up. In this respect, we find that there is an MHD generator ahead of the magnetic loop, where $\mathbf{E} \cdot \mathbf{J} \sim \mathbf{u} \cdot(\mathbf{J} \times \mathbf{B})<0$. Figure 11 shows the current flow lines that pass through the generator region and indicates that a generator current circuit is formed. We hence conclude that the MHD genertor ahead of the magnetic loop drives the current wedge. Also, global MHD simulations have suggested that the magnetic field dipolarization and the generation of sheared fields inside the loop are fundamental for the current wedge evolution (Raeder and McPherron, 1998; Janhunen and Palmroth, 2001). Hence, Fig. 12 shows the magnetic field lines that pass through the spatial points on the $y$-directional line at $x=0$ and $z=1.5$. We in fact see that when the fast reconnection jet collides with the loop at time $t \sim 42$, the field lines inside the loop are distinctly sheared, giving rise to fieldaligned currents to form the current wedge (Fig. 11).

\section{Summary and discussion}

Any theoretical model responsible for geomagnetic substorms must relevantly explain the well-known basic features that have been clarified by observations. Hence, the spontaneous fast reconnection model is applied to substorms and flares. It is demonstrated that the in-situ satellite ob-

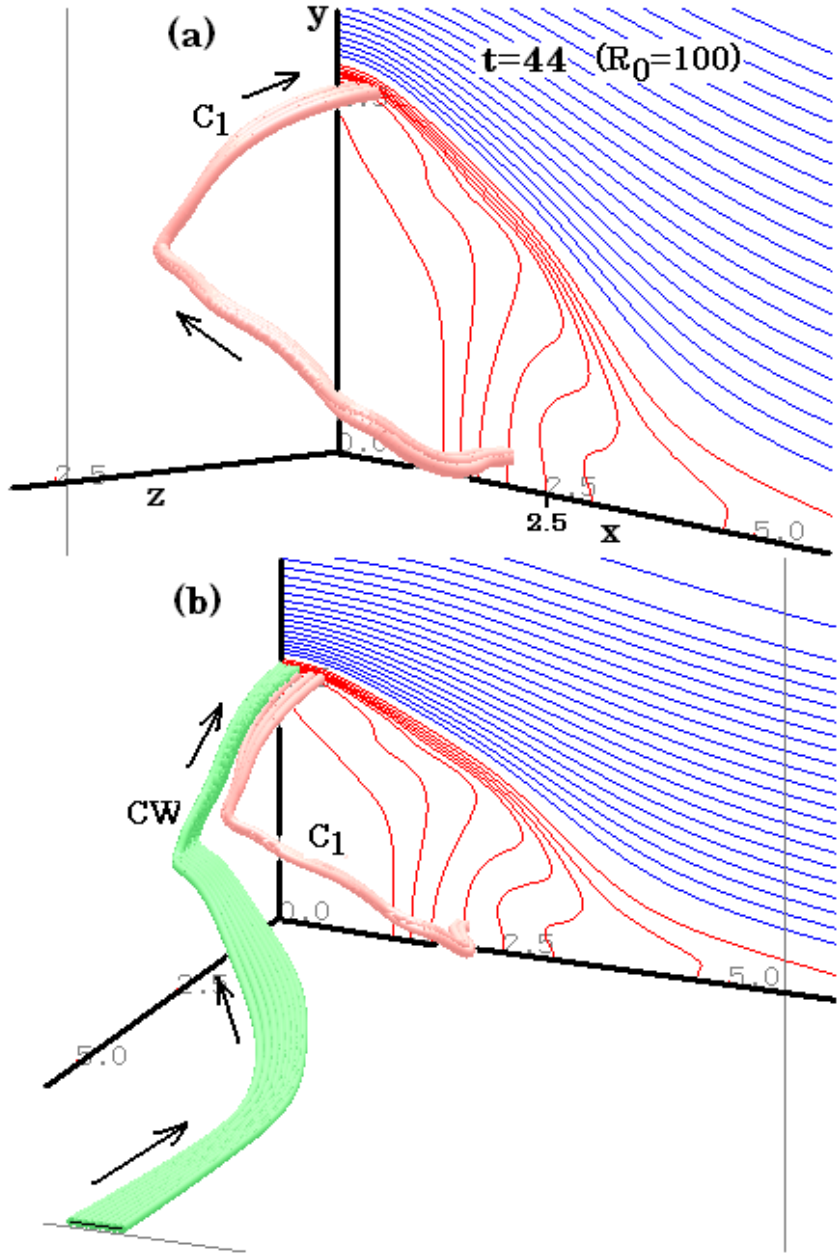

Fig. 11. (a) Current flow lines, denoted by $C_{1}$, that pass through the generator region ahead of the loop top, and in (b) the current wedge $(\mathrm{CW})$ that is formed simultaneously is also shown.

servations of TCR signatures can exactly be explained both qualitatively and quantitatively (Ugai and Zheng, 2006a, b); also, the 3-D fast reconnection jet collides with the magnetic 


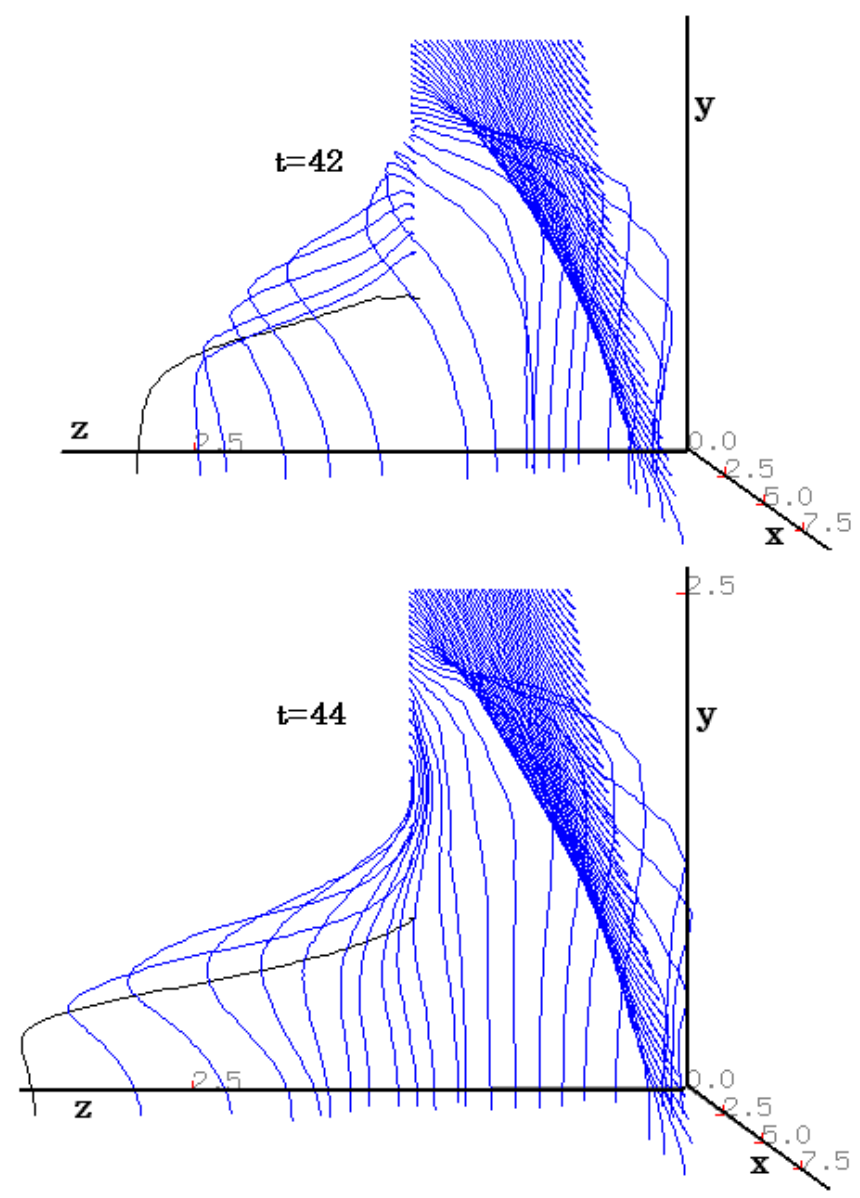

Fig. 12. Magnetic field lines that pass through the spatial points on the $y$-directional line at $x=0$ and $z=1.5$.

loop of closed field lines to cause drastic evolution of magnetospheric current wedge (Ugai and Kondoh, 2006; Ugai, 2007a). Recently, it is shown that satellite observations of earthward fast flows can well be explained by this theoretical model (Kondoh and Ugai, 2008). Regarding solar flares, it is shown that the chromospheric temperature is suddenly enhanced in the shape of two ribbons, and the resulting coronal flare loop is expanding outward; also, the definite chromospheric evaporation occurs because of the distinct joule heating in the chromosphere (Ugai, 2007b, 2008a). These are consistent with the well-known morphological features of two-ribbon flares.

Figure 13 schematically shows how the current wedge is driven by the MHD generator ahead of the magnetic loop. Although the complicated ionospheric boundary conditions are not pertinently considered, the underlying physical mechanism shown in Fig. 13 should be applicable to geomagnetic susbtorms. For instance, the MHD generator ahead of the loop will be crucial for sustaining field-aligned electric fields to accelerate nonthermal particles, although particle accelerations cannot be treated by MHD equations. Apparently,

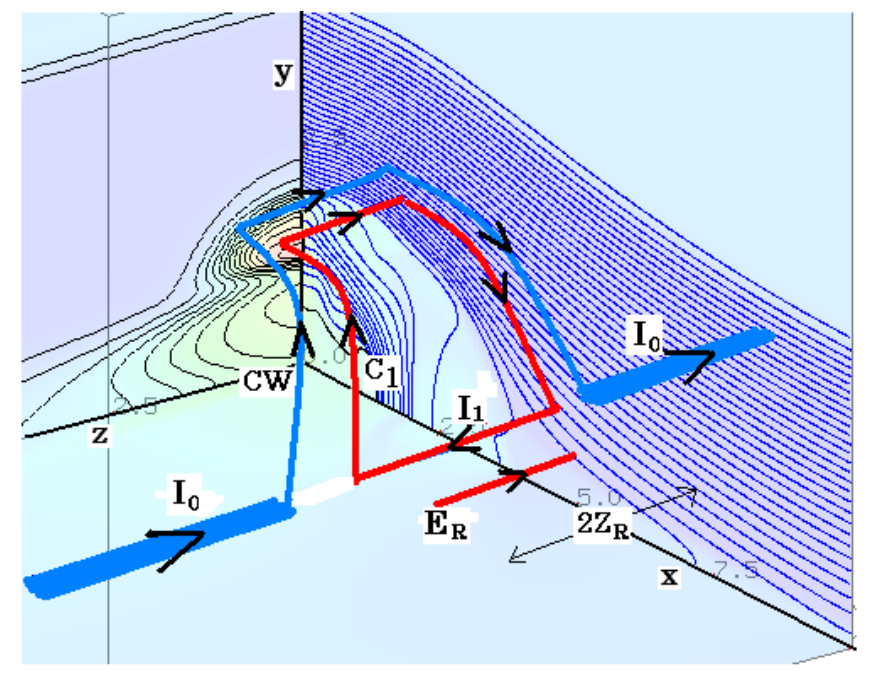

Fig. 13. Schematic drawing of the current circuit $C_{1}$, connected to the MHD generator ahead of the loop top; here, $\mathbf{E}_{\mathbf{R}}(\sim-\mathbf{u} \times \mathbf{B})$ is the reconnection electric field, $2 Z_{R}$ is the extent where the fast reconnection proceeds, $I_{0}$ is the initial coronal sheet current, and some part of $I_{0}$ becomes the current wedge, $C W$.

there are many similarities in the basic physics between solar flares and geomagentic substorms, so that we may conclude that both flares and substorms result from the same physical mechanism, i. e., the fast reconnection mechanism.

Acknowledgements. This work was supported by Grant-in-Aids for Creative Scientific Research "The Basic Study of Space Weather Prediction" (17GS0208, Head Investigator: K. Shibata) from the Ministry of Education in Japan, Mitsubishi Foundation, RISH of Kyoto University, and Solar-Terrestrial Environment Laboratory of Nagoya University.

Topical Editor R. Nakamura thanks two anonymous referees for their help in evaluating this paper.

\section{References}

Daughton, W., Scudder, J., and Karimabadi, H.: Fully kinetic simulations of undriven magnetic reconnection with open boundary conditions, Phys. Plasmas, 13, 072101, 2006.

Isobe, H., Miyagoshi, T., Shibata, K., and Yokoyama, T.: Filamentary structure on the sun from the magnetic Rayleigh-Taylor instability, Nature, 434, 478-481, 2005.

Janhunen, P. and Palmroth, M.: Some observational phenomena are well reproduced by our global MHD while others are not: Remarks on what, why and how, Adv. Space Res., 28, 1685-1691, 2001.

Karlicky, M.: Electron beam bombardment of the chromosphere in a flare loop with electric currents, Astron. Astrophys., 298, 913916, 1995.

Klimas, A. J., Uritsky, V. M., Vassiliadis, D., et al.: Reconnection and scale-free avalanching in a driven current-sheet model, J Geophys. Res., 109, A02218, doi:10.1029/2003JA010036, 2004. 
Kondoh, K. and Ugai, M.: Numerical studies on three-dimensional earthward fast plasma flows in the near-Earth plasma sheet by the spontaneous fast reconnection model, J. Geophys. Res., 113, A03S07, doi:10.1029/2007JA012707, 2008.

Lui, A. T. Y.: A multiscale model for substorms, Space Sci. Rev., 95, 325-345, 2001.

McPherron, R. L., Russel, C. T., and Aurbry, M. P.: Satellite studies of magnetospheric substorms on August 15, 1968. 9. Phenomenological model for substorms, J. Geophys. Res., 78, 31313149, 1973.

Petschek, H. E.: Magnetic field annihilation, in: AAS-NASA Symposium on the Physics of Solar Flares, NASA Spec. Pub., SP-50, 425-439, 1964.

Priest, E. R. and Forbes, T. G.: New models for fast steady state magnetic reconnection, J. Geophys. Res., 91, 5579-5588, 1986.

Raeder, J.: Global magnetohydrodynamics - A tutorial, in: Space Plasma Simulation, edited by: Buchner, J., Dum, C., and Scholer, M., Lecture Notes in Physics, 615, 212-246, 2003.

Raeder, J. and McPherron, R. L.: Global MHD simulations of the substorm current wedge and dipolarization, in: Substorms-4, p. 343-348, 1998.

Sharma, A. S., Nakamura, R., Runov, A., et al.: Transient and localized processes in the magnetotail: Review, Ann. Geophys., 26, 955-1006, 2008, http://www.ann-geophys.net/26/955/2008/.

Shibata, K.: Evidence of Magnetic Reconnection in Solar Flares and a Unified Model of Flares, Astrophys. Space Sci., 264, 129144, 1999.

Slavin, J. A., Smith, M. F., Mazur, E. L., et al.: ISEE 3 observations of traveling compression regions in the Earth's magnetotail, J. Geophys. Res., 98, 15 425-15 446, 1993.

Ugai, M.: Self-consistent development of fast magnetic reconnection with anomalous plasma resistivity, Plasma Phys. Controlled Fusion, 26, 1549-1563, 1984.

Ugai, M.: Global dynamics and rapid collapse of an isolated current-sheet system enclosed by free boundaries, Phys. Fluids, 29, 3659-3667, 1986.

Ugai, M.: MHD simulations of fast reconnection spontaneously developing in a current sheet, Computer Phys. Commun., 49, 185$192,1988$.
Ugai, M.: Computer studies on development of the fast reconnection mechanism for different resistivity models, Phys. Fluids B, 4, 2953-2963, 1992.

Ugai, M.: Computer studies on the spontaneous fast reconnection model as a nonlinear instability, Phys. Plasmas, 6, 1522-1531, 1999.

Ugai, M.: Conditions for drastic evolution of magnetospheric current wedge, Phys. Plasmas, 14, 062902, 2007a.

Ugai, M.: Modeling of two-ribbon flares by the fast reconnection mechanism, Phys. Plasmas, 14, 102904, 2007b.

Ugai, M.: Impulsive chromospheric heating of two-ribbon flares by the fast reconnection mechanism, Phys. Plasmas, 15, 032902, 2008a.

Ugai, M.: The evolution of fast reconnection in a three-dimensional current sheet system, Phys. Plasmas, 15, 082306, 2008b.

Ugai, M. and Tsuda, T.: Magnetic field-line reconnexion by localized enhancement of resistivity. Part 1 . Evolution in a compressible MHD fluid, J. Plasma Phys., 17, 337-356, 1977.

Ugai, M. and Zheng, L.: Conditions for the fast reconnection mechanism in three dimensions, Phys. Plasmas, 12, 092312, 2005.

Ugai, M. and Zheng, L.: Modeling of traveling compression regions in the Earth's magnetotail by the spontaneous fast reconnection model, Phys. Plasmas, 3, 032901, 2006 a.

Ugai, M. and Zheng, L.: Parametric studies on traveling compression regions observed in the Earth's magnetotail, Phys. Plasmas, 13, 062906, $2006 \mathrm{~b}$.

Ugai, M. and Kondoh, K.: Evolution of magnetospheric current wedge by the spontaneous fast reconnection model, Phys. Plasmas, 13, 102903, 2006.

Uzdensky, D. A.: Petschek-like reconnection with current-driven anomalous resistivity and its application to solar flares, Astrophys. J., 587, 450-457, 2003.

Vasyliunas, V. M.: Theoretical models of magnetic field line merging, Rev. Geophys. Space Phys., 13, 303-336, 1975.

Yokoyama, T. and Shibata, K.: What is the condition for fast magnetic reconnection, Astrophys. J., 436, L197-L200, 1994. 